\title{
MEMORIAS DE LA INTIMIDAD: LA VOZ DE LAS POETAS SEFARDÍES ANTE LAS TRANSFORMACIONES DEL SIGLO XX
}

\author{
Elisa Martín Ortega \\ CSIC, Madrid
}

Los procesos de construcción de la memoria histórica reflejan las particularidades de los colectivos que los llevan a cabo: su función social y su visión del pasado. La elaboración de una memoria propia de los acontecimientos del siglo xx representa un interesante ejemplo de la irrupción de las mujeres en la vida pública (a través de manifestaciones políticas, artísticas o sociales), y también de su contribución a una privatización de lo público.

La memoria, tal como se ha configurado tras la Segunda Guerra Mundial y la tragedia del Holocausto, constituye un terreno especialmente fecundo para el estudio de los puntos de confluencia entre lo público y lo privado. Partiendo de las teorías de Walter Benjamin, hemos asistido al rescate de su aspecto sentimental e íntimo, dignificándolo y considerándolo un modo específico de conocimiento histórico. La actividad literaria ha ido alcanzando, desde entonces, un papel cada vez más relevante en la elaboración de la memoria, sin restarle por ello contenido político. El filósofo Reyes Mate escribe al respecto:

La memoria es desde luego privada. Si al Proust de Du côté de chez Swann le viene encima de sopetón toda su vida vivida, es porque la resucita la modesta magdalena que acaba de mojar en el té. Ahora bien, si lo que se pretende decir

\footnotetext{
${ }^{1}$ Este artículo ha sido realizado en el marco del proyecto de investigación FFI2012-31625: "Los sefardíes ante sí mismos y sus relaciones con España. Hacia la recuperación de un patrimonio cultural en peligro", del Ministerio de Economía y Competitividad.
} 
es que esa memoria no puede ser pública o política, hay que responder que la memoria es privada y también pública. (Reyes Mate 2009: 161)

En este artículo trataremos de analizar tal circunstancia desde el punto de vista de las mujeres sefardíes del siglo xx, estudiando algunas composiciones de tres poetas que escriben en judeoespañol: Margalit Matitiahu, Clarisse Nicoïdski y Mijal Held. En sus obras se refleja la nostalgia ante el desmoronamiento de las comunidades sefardíes tradicionales (como consecuencia de la emigración, los cambios socio-culturales, y muy especialmente el exterminio nazi).

Las comunidades sefardíes ${ }^{2}$ fueron fundadas por los judíos expulsados de la Península Ibérica a finales de la Edad Media. Los exiliados y sus descendientes se instalaron principalmente en territorios pertenecientes al antiguo Imperio Otomano (Turquía, Grecia, Balcanes, Norte de África), y su lengua de comunicación era el judeoespañol ${ }^{3}$. A lo largo de sus más de quinientos años de historia, las comunidades desarrollaron una importante actividad religiosa, cultural y económica. En la sociedad sefardí tradicional, las mujeres ocupaban el espacio de lo privado y de lo familiar y lo doméstico, así como el ámbito de la cultura oral ${ }^{4}$, mientras que la vida pública y la cultura escrita pertenecían al campo de lo masculino.

A lo largo de la primera mitad del siglo xx se produjeron importantes cambios que transformaron la vida de los sefardíes y el papel que había sido asignado a la mujer ${ }^{5}$. En primer lugar, el judeoespañol comenzó a dejar de utilizarse y de transmitirse en la familia a causa de múltiples factores: la modernización de las sociedades y la llegada de los nacionalismos, que impulsó el uso de las lenguas mayoritarias de los países donde vivían los sefardíes; la propia disolución de las comunidades y el abandono de la vida tradicional tras los cambios en la educación y la introducción de escuelas occidentales, sobre todo las de la Alliance Israélite Universelle, que tuvieron una gran difusión entre los sefardíes, y desde las que se propugnaba una evolución de las costumbres y una mayor apertura a la cultura y la ciencia europeas; ${ }^{6}$ el cambio paulatino en el papel de la mujer, que había sido durante siglos la encargada de conservar y transmitir la lengua judeoespañola y su patrimonio folklórico y de literatura oral.

2 Para mayor información sobre la historia y la cultura de los sefardíes ver Díaz Mas (2006) y Rodrigue y Benbassa (2004).

${ }^{3}$ Una panorámica útil sobre el judeoespañol se puede encontrar en Lleal (1992). Sobre la formación del judeoespañol tras la expulsión ver Penny (2000: 174-193). Para las distintas variedades de la lengua sefardí: Quintana (2006) y (2010: 33-54). Distintos aspectos de sociolingüística han sido tratados en Bunis (1982: 41-70) y Filipovic y Vucina Simovic (2010: 259-270). Sobre la decadencia del judeoespañol ver Vidal Séphiha (1977), Harris (1994) y Schmid (2007).

${ }^{4}$ Para el papel de las mujeres como preservadoras y transmisoras de la cultura oral sefardí hasta el mismo siglo xx puede verse Seroussi (2003: 195-214); Cohen (1995: 181-200); Díaz-Mas (2009: 81-101), (2008: 255-266) y (2007: 187-200); Havassy (2010: 247-256).

${ }^{5}$ Sobre la mujer sefardí y los cambios en su rol tradicional: Díaz-Mas, Ayala y Barquín (2009: 2239); Ayala (2006: 45-67) y (2008: 144-155); Quintana (2009: 113-139).

6 Para ampliar información acerca de los cambios en la educación: Rodrigue (1983: 263-286) y (1990). 
Si bien estas transformaciones supusieron una evolución en la vida de las comunidades y debilitaron la presencia del judeoespañol, hay que destacar que se trataba de una lengua todavía ampliamente empleada entre los sefardíes en casi todos los órdenes de la vida. Sin embargo, tras la Segunda Guerra Mundial y el exterminio de los judíos europeos, la situación cambió drásticamente. Hubo comunidades enteras (como la de Salónica, en Grecia) cuyos miembros fueron deportados masivamente y quedaron arrasadas. El impacto que tuvo el Holocausto en la vida y las conciencias de los sefardíes (incluso de aquellos que, por los países donde residían, no lo sufrieron directamente) fue enorme. Tras el drama, muchas de las comunidades se dispersaron, algunos de sus miembros emigraron a Israel o a otros países (como Francia, Estados Unidos o Argentina), y la cultura tradicional, así como el uso cotidiano de la lengua judeoespañola, desaparecieron casi por completo.

Las mujeres, que habían tenido un papel muy limitado en la vida pública y en la creación literaria en judeoespañol hasta bien entrado el siglo $\mathrm{xx}^{8}$, construyen por primera vez, en el momento del ocaso de la vida tradicional y de la propia lengua judeoespañola, una voz propia. Se convierten, así, en testigos de la extinción de sus comunidades, de las consecuencias del Holocausto, de la guerra y la emigración forzosas 9 . Sus composiciones ofrecen una particular visión de tales acontecimientos, en la que lo doméstico, y el patrimonio cultural recibido de sus madres y abuelas ocupa un lugar fundamental. A través de sus poemas es posible realizar un recorrido por la memoria de la intimidad, proponiendo una nueva mirada sobre el pasado sefardí y renovando el interés por su recuerdo; mirada que, al mismo tiempo, refleja las convulsiones políticas, sociales y culturales que afectaron a las comunidades durante la segunda mitad del siglo xx.

¿Por qué circunscribimos nuestro corpus a la poesía, un género que podríamos considerar profundamente subjetivo y poco dado a reflejar lo histórico y lo memorístico? En primer lugar, porque tal como señalan Riaño López y Marcos Casquero (2001: 169), "cuando hablamos de literatura sefardí contemporánea, hoy en avanzado proceso de extinción, nos estamos refiriendo solo a un género, el de la poesía de autor, pues casi nada nuevo se ha compuesto en español sefardí en época cercana a nuestros días que no sean poemas". Más allá de esta circunstancia, el paso operado de la "historia" a la "memoria" permite a la poesía judeoespañola escrita por mujeres dar un testimonio de lo íntimo y convertirlo, de inmediato, en un hecho público y político. Al mismo tiempo, la escritura de estas mujeres constituye un encomiable esfuerzo por rescatar la propia lengua judeoespañola y convertirla en el único y verdadero vehículo de transmisión de la memoria.

\footnotetext{
7 Sobre la literaratura sobre el Holocausto escrita por sefardíes: Refael (2003); Santa Puche (2003); Lévy (1989).

8 Una panorámica sobre la literatura sefardí puede verse en Romero (1992).

${ }^{9}$ Para mayor información sobre las memorias de mujeres sefardíes ver Romeu Ferré (2008: 101120).
} 


\section{LA VOZ DE LA MADRE}

La voz de los antepasados, y muy especialmente la de la madre, es el principal punto de arranque de los esfuerzos de las poetas sefardíes de la segunda mitad del siglo xx por rescatar su pasado. Las autoras, que normalmente han nacido en países de la segunda diáspora sefardí10, y por tanto han crecido en entornos en los que la lengua y la cultura sefardíes habían quedado relegados al ámbito doméstico, reconocen en el legado de sus madres un símbolo de esa tradición en decadencia. De hecho, en más de una ocasión, es precisamente la muerte de la madre lo que motiva el comienzo de la actividad poética en judeoespañol. Clarisse Nicoïdski (Lyon, 1938-1996), novelista en francés y, tardíamente, poeta en judeoespañol, explica del siguiente modo las razones que le llevaron a esta aventura literaria:

La muerte de mi madre fue una grande conmoción. Además comprendí que con ella se iba definitivamente algo de esta lingua de mi infancia, y que para nuestra generación la muerte de nuestros mayores significaba la muerte de un lenguaje.

En esa lingua se hallaba el amor de mi madre, nuestra complicidad y nuestras risas.

Así me atreví a escribir estos poemas para que quede la empresa de su voz. Cada vez que terminé un libro en francés me dediqué a escribir en muestru spaniol algo como un canto.

No se nada de religión, o cerca nada, ma quisiera que estas palabras en la lingua perdida sean para ella, mi madre, como un kadish, repetido a menudo. (Nicoïdski 1999: 37) $)^{11}$

También Margalit Matitiahu (Tel Aviv, 1935, de padres de Salónica) refiere una experiencia similar. En uno de sus libros más célebres, Kurtijo kemado (TelAviv, Eked, 1988), la autora recrea la vivencia de un viaje a las comunidades judías de Grecia que realizó a mediados de los años 80 con un grupo de descendientes de supervivientes del Holocausto. Tal como ella misma explica, decidió participar en este recorrido "con la intención de rendir homenaje al recuerdo de su madre, que había fallecido unos pocos meses antes" (Refael 2009: 168). El primer poema del libro lo muestra con claridad:

\section{ANTES DE ARIVAR A SALONIKI}

Antes de la partensia al portal de tu chikes

Vine madre mia

A batir enriva la piedra de tu kaza eternal

\footnotetext{
10 Se denomina segunda diáspora a la de los sefardíes que, a lo largo del siglo xx, abandonaron los territorios donde sus antepasados se habían instalado tras la expulsión de España (fundamentalmente el Norte de África, Grecia, Turquía y los Balcanes), y emigraron a países como Israel, Estados Unidos, Argentina o Francia, con la consecuente disolución de las comunidades tradicionales.

${ }^{11}$ Entiéndase kadish: oración fúnebre judía, que el huérfano debe rezar todos los días durante un año tras la muerte de su padre. Pasado este tiempo, se considera que ha terminado el periodo de duelo.
}

176 | Pasavento. Revista de Estudios Hispánicos, vol. II, n. ${ }^{\circ} 1$ (invierno 2014), pp. 173-185, ISSN: 2255-4505 
I dezirte ke me vo

Ande se crio tu alma

Ande tu padre planto en ti simiente de poezia.

Me aserko de tu sufriensa kayada

I de las piedras ke no tienen luz

Ma todo no fue en vano

Vo a murmurear en tu oreja de tierra. (Matitiahu 1998: 9) ${ }^{12}$

La visita a la tumba de la madre revela el propósito del viaje: llegar "al portal de tu chikes", a ese lugar misterioso donde la madre pasó su infancia. La poesía se concibe como un legado que pasa de generación en generación, al igual que la lengua, tal como se expresa en el verso "Ande tu padre planto en ti simiente de poezia". Margalit Matitiahu sale en busca de los lugares de la memoria, que no son otros que los escenarios de la niñez de su madre, único puente con el pasado. Por ello, antes de iniciar el viaje a Salónica, se produce este encuentro, soñado e imposible. La declaración de intenciones culmina en el verso final: "Vo a murmurear en tu oreja de tierra"; los poemas que componen Kurtijo kemado no serían sino ese murmullo que con el que la autora trata de cavar en la tierra. No aparece en la obra la imagen de la elevación, tan recurrente cuando se trata de establecer relaciones con los muertos; por el contrario, asistimos a un constante intento de ahondamiento, penetración, que da idea de lo arduo de la tarea, de la profundidad del sufrimiento, pero también del enraizamiento necesario para llevarla a cabo.

La memoria del dolor recorre el libro. Ya en este primer poema, dedicado a la madre, Matitiahu escribe: "Me aserko de tu sufriensa kayada". Ese es el verdadero propósito de su escritura: rescatar del silencio los padecimientos y la muerte de sus antepasados $y$, de forma más general, de los sefardíes durante el Holocausto. El poema "En el kamino de Athena a Larisa", en el que se evoca la deportación de los judíos griegos en los trenes hacia los campos de exterminio, termina con los siguientes versos:

Kon la marka de dolor en el kuerpo,

Komo la mezuza

Bezar, bezar i Akodrar (Matitiahu 1998: 13)

La mezuzá es un receptáculo que se adhiere a la jamba derecha de las puertas en las casas judías. En su interior se encuentra un pergamino enrollado con unos versículos de la Biblia (Deuteronomio 6:4-9 y 11:13-21) en los que se insiste en la necesidad de inscribir las palabras en el cuerpo y el alma, cual muestra de fidelidad y de memoria, al mismo tiempo que se insta a recordar y transmitir la religión y la cultura judía a las generaciones venideras. Los judíos practicantes suelen "besar" la mezuzá con su mano al pasar junto a ella, tanto a la entrada como a la salida de la casa. Matitiahu retoma esta imagen, símbolo de lealtad a la tradición, de confianza y protección, y la traslada a la desgracia

12 Entiéndase chiques: niñez, infancia. 
de los deportados y el compromiso con su memoria. Tanto los muertos como los supervivientes Ilevan "la marka de dolor en el kuerpo". Y es precisamente esa marca la que dota de legitimidad y de verdad al poema. La obligación de recordar y grabar en el propio cuerpo las palabras de la mezuzá, de amarlas a través del "beso" o caricia, se ha convertido ahora en un deber de memoria que tiene que ver con lo íntimo y con lo físico, y que basa su autenticidad en la experiencia del sufrimiento. Reyes Mate vincula este descubrimiento a la elaboración de la memoria del Holocausto:

Si Auschwitz es singular, si podemos hablar de que hay un antes y un después, es porque ahí se pone de manifiesto algo que siempre había acompañado a la historia pero que hasta ese momento había conseguido insensibilizarse o camuflarse: el sufrimiento del otro. [...] Se había camuflado tanto que habíamos llegado a pensar que la verdad casa con la objetividad, la impasibilidad, la apatía, la neutralidad, pero no con la experiencia del sufrimiento. (Reyes Mate 2009: 170)

Desde esta perspectiva, la creación poética es fiel compañera de la memoria, pues es precisamente a partir de su compromiso con el universo de lo íntimo y su elaboración del sufrimiento silenciado como es posible aprehender y transmitir la verdad del pasado. Entendemos, entonces, la memoria como una peculiar actividad hermenéutica: "la de hacer visible lo invisible" (Reyes Mate 2009: 167). Y, en el caso de nuestras poetas, el testigo dejado por el legado de la madre es recogido por el deseo de habitar los lugares del pasado.

\section{ESPACIOS DE LA VIDA PRIVADA}

Las ciudades en las que vivieron los sefardíes hasta la segunda mitad del siglo $x x$ y de las que emigraron o fueron deportados, son los escenarios predilectos de los poemas de estas mujeres sefardíes. En su tratamiento destaca el predominio de la nostalgia y la atención a los espacios de la vida cotidiana. Las calles y las casas se convierten así en ruinas cuyo principal valor es su capacidad para evocar los recuerdos.

Mijal Held, nacida en Israel en la década de los sesenta, investigadora y profesora de la Universidad Hebrea de Jerusalén, y fundamentalmente escritora en hebreo, compone un poema en el que la voz poética realiza un recorrido a través de varias ciudades que fueron de especial importancia para las comunidades sefardíes. Se propone un viaje en el espacio, pero también en el tiempo:

Irme kero, madre, a Salonik,

A kantar sus viejas romansas

Pedridas.

I a Monastir kero ir,

A riyir kon sus kriaturas

En lingua

Dulse. 


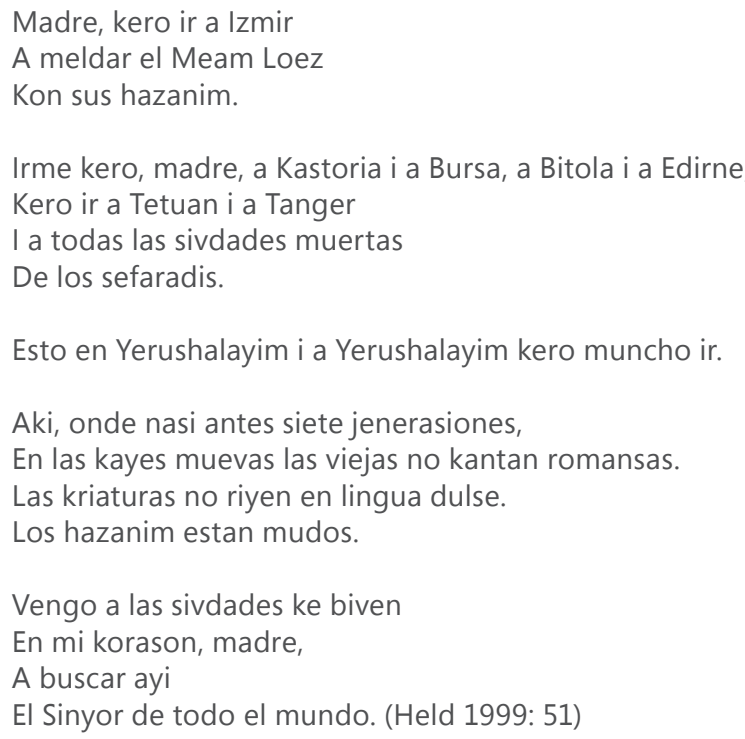

Los lugares de la memoria viven en el corazón: son paisajes reales, ahora muertos (desde el punto de vista de la cultura sefardí), pero capaces de convocar la vida. Las calles y las casas recuerdan "viejas romansas", "kriaturas", "hazanim" que leen el Meam Loez, y sobre todo la "lingua dulse", en clara alusión al judeoespañol que ya no se escucha. Son significativas las referencias constantes a la madre, a los niños, a la lengua, y también a esos romances perdidos: "en las kayes muevas las viejas no kantan romansas". La poesía tradicional sefardí, de transmisión oral, tuvo en la memoria de las mujeres su último reducto: ellas fueron las últimas guardianas de este patrimonio musical y literario, que acompañaba a los miembros de las comunidades en gran parte de sus celebraciones y en los momentos esenciales del ciclo vital. Jerusalén, la última ciudad nombrada, es un espacio doble, por eso Held escribe: "Esto en Yerushalayim i a Yerushalayim kero muncho ir", en una aparente paradoja. La ciudad actual, en la que vive la autora, se contrapone al hogar soñado que fue habitado por el judeoespañol un día.

En el poema de Mijal Held encontramos ecos de una canción tradicional sefardí, compuesta a partir de dos coplas ${ }^{13}$ en las que se expresa la nostalgia de Sión, el deseo de regresar a Jerusalén y restaurar el Templo destruido. Presentamos a continuación una versión cantada de Jerusalén, recogida en 1960 por Israel J. Katz y editada por Paloma Díaz-Mas:

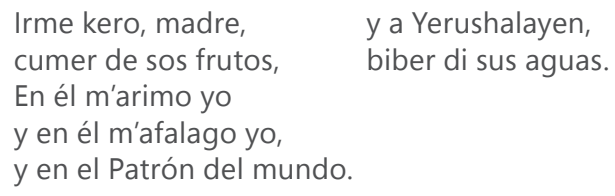

${ }^{13}$ Las coplas están recogidas en Hemsi (1995: 158a-b, 159). 


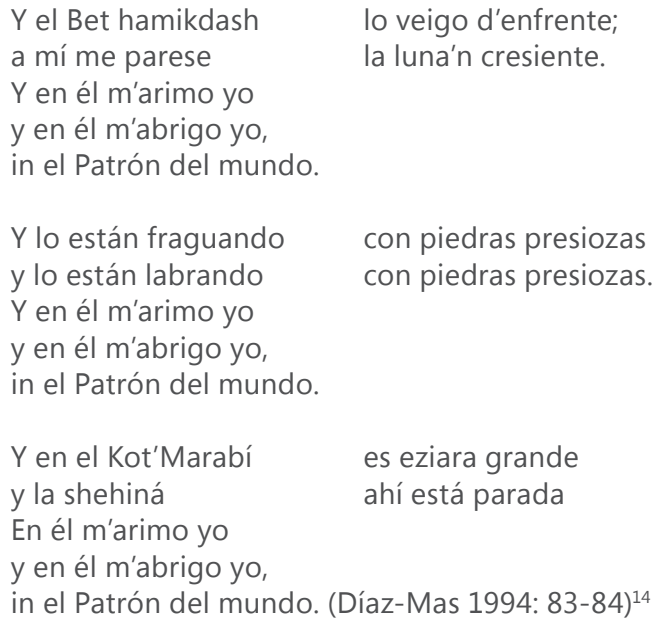

La canción recoge el deseo del pueblo judío de regresar a su patria perdida, presente entre los sefardíes a lo largo de la historia. Aparece la esperanza en la llegada de los tiempos mesiánicos, simbolizada en la restauración del Templo. Mijal Held toma este poema tradicional y lo recrea de forma desencantada, confrontando el rico pasado sefardí con un presente desierto. El motivo del viaje y la apelación a la madre "Irme kero, madre..." están tomados directamente de la canción tradicional.

Un movimiento similar lo encontramos en el poema de Margalit Matitiahu que lleva por título "Saloniki":
SALONIKI
(En la kaye ke-se engrandisio mi madre)
La kaye paresia intchirse de mis ondos sintimientos,
En kaminando i buscando mi viejo nombre.
Las solombras de la tarde
Empesavan a kayer sobre las kazas.
Las ventanas seradas paresian metersen
En una gera muda kontra el tiempo pasado.
Mezo las memorias plantadas en mi por mi madre
Via la kaza ke supito se enchekisiya
Asta tokar la tierra del kurtijo
Ande las bozes de lo pasado kedaron en el aver.

\footnotetext{
${ }^{14}$ La grafía ha sido modificada y se ha adoptado la de la revista Aki Yerushalayim. Entiéndase m'arimo: me acojo; m'afalago: me consuelo; Patrón (del mundo): dueño, aquí se refiere a Dios; Bet hamikdash: la casa santa, el Templo de Jerusalén; máabrigo: me protejo; Kot'Marabí: muro occidental o muro de las lamentaciones, el único resto que queda del Templo de Jerusalén; eziara: romería, peregrinación; shehiná: presencia divina en el mundo, que según la tradición mora en el Templo destruido.
} 
Y sintia la prononsiasion de un nombre

Komo una kampana

Kunandose en el tiempo i disiendo

Thessaloniki, Odos Theoyenos Harisis 59. (Matitiahu 1998: 17) $)^{15}$

La emoción ante la cercanía de la casa de la madre, que la poeta no había visitado hasta entonces, canaliza una "gerra muda kontra el tiempo pasado". El poema está atravesado por la idea de mecer, de acunar, en una sugerente metáfora: "Mezo las memorias plantadas en mi por mi madre". La memoria es una semilla que se recibe, y los lugares del pasado la hacen crecer. El movimiento es hacia lo pequeño ("la kaza se enchekisiya"), pues solo esa vuelta a lo mínimo, a la niñez, a lo aparentemente insignificante puede convocar a las voces y la tierra del "kurtijo", el espacio por excelencia de la intimidad femenina en las vecindades sefardíes. La dirección de la casa parece convertirse, en el último verso del poema, en una palabra mágica, que Matitiahu define como "una kampana kunandose en el tiempo". Acunar y mecer son los gestos que permiten el rescate de la memoria y el pasado, en una última referencia a la relación entre madre e hija, enmarcada ahora en el contexto del hogar perdido.

\section{Mirar el mundo con los ojos de los olvidados}

Ahora bien, ¿hasta cuándo podemos hablar de memoria? ¿En qué medida estas poetas sefardíes pueden elaborar la memoria de un mundo en el que ya no han vivido? Reyes Mate vuelve a esbozar una respuesta:

La fragilidad de la memoria se debe a que en un momento determinado el pasado vivido deje de ser traditus entregado a un colectivo que lo recibe. Al romperse la traditio cesa la memoria. Según esta explicación, cuando llega la hora de la historia, se cierra el tiempo de la memoria. Cuando desaparecen los testigos, deberíamos dejar de hablar de memoria. [...] Pero es muy difícil precisar cuándo se rompe el vínculo entre el pasado y el presente porque siempre quedan huellas, muchas veces ocultas, de modo que en un determinado momento el presente declara verse reflejado en ellas. (Reyes Mate 2009: 160)

Margalit Matitiahu, en otro poema de Kurtijo kemado, da fe de la expansión de la memoria más allá de su territorio natural: el del recuerdo individual e intransferible. Leamos sus últimos versos:

\section{LA MEMORIA}

$[\ldots]$

La memoria se espande, deviene velas palpando.

Yo me encolgo en ellas

\footnotetext{
${ }^{15}$ Entiéndase engrandesio: creció; enchekisiya: empequeñecía; kurtijo: patio interior que solían tener las casas de vecindad, donde se hacía vida en común; representa el espacio por excelencia de las relaciones entre las mujeres de las comunidades sefardíes tradicionales; aver: aire.
} 


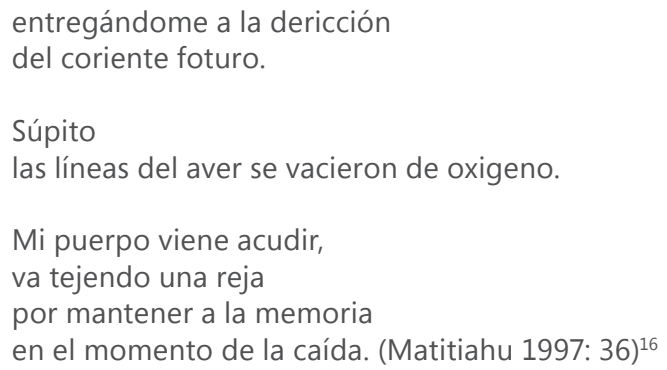

La voz poética se entrega a la travesía de la memoria, que es al mismo tiempo la corriente del futuro. "La memoria se espande" $y$, en una metáfora marina, se convierte en las velas a las que se agarra la poeta para continuar su camino hacia el porvenir. Así, el pasado es una presencia que acompaña, que empuja hacia delante. En los versos finales es el cuerpo el que realiza el rescate último de la memoria: crea una red que la salva del precipicio.

¿Qué es lo que ofrece esa memoria recreada al futuro? Su lugar, como ya se vio en un poema anterior, se encuentra bajo tierra: ¿en el territorio de los muertos? Sí y no. El rescate del pasado sefardí exige a estas poetas adentrarse en el universo de los antepasados (sobre todo a través de la figura-guía de la madre), recorrer los espacios de la vida perdida, aunque sea entre ruinas, $y$, finalmente, sumergirse en las profundidades de una tierra que, a pesar de las desdichas, esconde un tesoro. Veamos otro poema de Matitiahu:

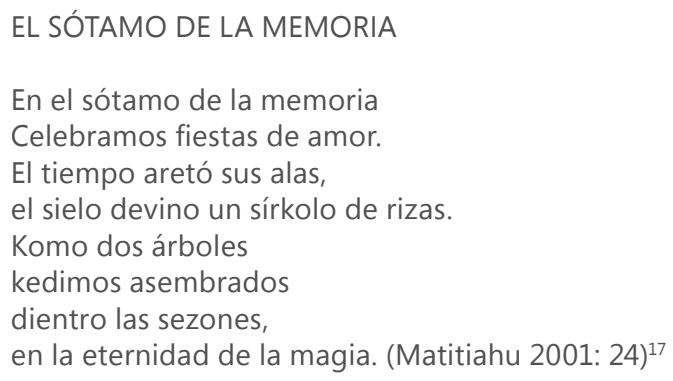

Al final de un recorrido descendente, que va internándose en lo profundo y recogiendo el dolor del pasado, ¿qué sucede?: "Celebramos fiestas de amor". La voz poética se asombra y vislumbra, ya a su lado, "la eternidad de la magia"; una promesa de comunión con lo perdido que sitúa el centro de la vida y la esperanza en la cercanía con lo aniquilado. Clarisse Nicoïdski comparte tal paradoja en el poema que abre su segunda obra, "Caminus di palavras":

\footnotetext{
${ }^{16}$ Entiéndase dericción: dirección; aver: aire.

17 Entiéndase aretó: paró; sezones: razones.
} 


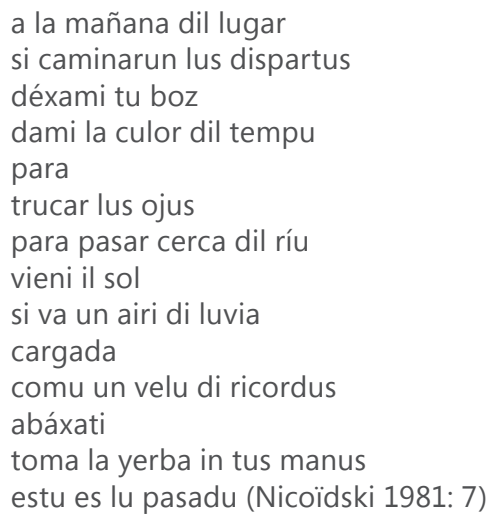

La presencia a la que se refiere la voz poética en segunda persona es, una vez más, la memoria, encarnada aquí en la lengua judeoespañola, el idioma de los antepasados, y en la imagen de la propia muerte (a la que se contrapone la mención a "lus dispartus"). La poeta la insta a que le entregue su voz, la única capaz de cambiar lo que ven los ojos, pues conoce y ofrece "la culor dil tempu". La humedad y el río representan el pasado vivo, incandescente: esa lluvia que deja tras de sí "un velo di ricordus" sobre la hierba. Finalmente la hierba, cercana a la tierra pero mojada por la lluvia, es la encargada de transmitir la huella de lo desaparecido.

Esta nueva relación con la realidad y con el tiempo presente, que aporta creatividad y salva del silencio a lo pasado, no deja de ser un regalo de la memoria: consiste en mirar el mundo con los ojos de los olvidados. En esa mirada hay dolor, pero también fascinación y belleza. Luigi Pirandello, en un relato titulado "Coloquio con los personajes", reflexiona acerca de la relación entre los vivos y los muertos a raíz de la desaparición de su madre. En un momento la madre, que habla desde el pensamiento del personaje principal, le dice: "Guarda le cose anche con gli occhi di quelli che non le vedono più! Ne avrai un rammarico, figlio, che te le renderà più sacre e più belle". (Pirandello 1980: 1153)

Este es, quizá, el máximo valor de la memoria: permitir una mirada desde el lugar de los desaparecidos, rescatando su legado para volver a habitar la realidad con sus ojos: unos ojos silenciosos pero capaces de convocar a su alrededor la vida. El recorrido que las poetas sefardíes evocadas hacen por los paisajes interiores y las palabras de sus antepasados conducen, una vez traspasado el dolor, a esas "fiestas de amor" a las que se refería Margalit Matitiahu. Así nos sugieren que la promesa se ha cumplido, que es posible, sin abandonar el presente, mirar el mundo desde el lugar de los olvidados. 


\section{OBRAS CITADAS}

Ayala, Amor (2008): "La instruksion es el mas ermozo afeite por la mujer. Una conferencia de David Fresco sobre la nueva mujer sefardí (Estambul, 1929)". En: Sefárdica, n. ${ }^{\circ}$ 17, pp. 144-155.

Ayala, Amor (2006): "«La mujer moderna» por Y. A. Basat (La Alvorada, Ruse 1899): la mujer sefardí y sus deberes en la nueva sociedad". En: Miscelánea de estudios árabes y hebraicos. Sección hebreo, n. ${ }^{\circ}$ 55, pp. 45-67.

Bunis, David M. (1982): "Types of nonregional variation in Early Modern Eastern Spoken Judezmo". En: International Journey of Sociology of Language, n. ${ }^{\circ} 37$, pp. $41-70$.

Cohen, Judith R. (1995): "Women's Role in Judeo-Spanish song traditions". En: Maurice Sacks (ed.): Active Voices. Women in Jewish Culture. Chicago, University of Illionis, pp. 181-200.

Díaz-Mas, Paloma, Ayala, Amor, y Barquín, Amelia (2009): "La incorporación de las mujeres sefardíes a la esfera pública en el paso del siglo xIx al xx". En: Actas del primer congreso internacional Las mujeres en la esfera pública. Filosofía e historia contemporánea. Madrid, C.E.R.S.A., pp. 22-39.

Díaz-Mas, Paloma (2009): "Folk Literature among Sephardic Bourgeois Women at the Beginning of the Twentieth Century". En: European Journal of Jewish Studies, vol. 3, n. ${ }^{\circ} 1$, pp. 81-102.

(2008): "Las mujeres sefardíes del Norte de Marruecos en el ocaso de la tradición oral". En: El Presente. Studies in Sephardic Culture, n. ${ }^{\circ} 2$, pp. 255-266.

(2007): "Cuadernos de mujeres: el cuaderno de Clara Benoudis y otras colecciones manuscritas de cantares tradicionales sefardíes". En: Kelly Benoudis (ed.): Romances de Alcácer Quibir. Lisboa, Edições Colibri-Centro de Estudos Comparatistas, pp. 187-200.

(2006): Los sefardíes: Historia, lengua y cultura. Barcelona, Riopiedras (4a ed.).

Díaz-Mas, Paloma (ed.) (1994): Poesía oral sefardí. Ferrol, Esquío.

Filipovic, Jelena, y Simovic, Ivana Vucina (2010): "La lengua como recurso social: el caso de las mujeres sefardíes de los Balcanes". En: Paloma Díaz-Mas y María Sánchez Pérez (eds.): Los sefardíes ante los retos del mundo contemporáneo. Identidad y mentalidades. Madrid, CSIC, pp. 259-270.

Harris, Tracy K. (1994): Death of a language: The history of Judeo-Spanish. Newark. University of Delaware Press.

Held, Mijal (1995): "Irme Kero, madre". En: Aki Yerushalayim, n. ${ }^{\circ} 61$, p. 51.

Hemsi, Alberto (1995): Cancionero sefardí. Jerusalén, The Jewish Music Research Centre, The Hebrew University of Jerusalem, 158a-b, 159.

Lévy, Isaac Jack (ed.) (1989): Sephardic poetry of the Holocaust. Chicago, University of Illiois Press.

Lleal, Coloma (1992): El judezmo. El dialecto sefardí y su historia. Barcelona, Universidad de Barcelona.

Matitiahu, Margalit (2001): Bozes en la Shara. Cuenca, El toro de barro.

(1998): Kurtijo kemado. Tel Aviv, Eked. (1997): Matriz de luz. Tel Aviv, Tag.

184 | Pasavento. Revista de Estudios Hispánicos, vol. II, n. ${ }^{1}$ (invierno 2014), pp. 173-185, ISSN: 2255-4505 
Nicoïdski, Clarisse (1999): "Palabras introductorias". En: Dina Rot (ed.): Una manu tumó l'otra. Madrid, El Europeo.

(1981): "Caminus di palavras". En: Poesía X. Madrid, Editora Nacional, pp. 6-14.

Penny, Ralph (2000): "Variation in Judeo-Spanish". En: Variation and change in Spanish. Cambridge, Cambridge University Press, pp. 174-193.

Pirandello, Luigi (1980): Novelle per un anno. Vol. III, tomo II. Milán, Mondadori.

Quintana, Aldina (2010): "El judeoespañol, una lengua pluricéntrica al margen del español". En: Paloma Díaz-Mas y María Sánchez Pérez (eds.): Los sefardíes ante los retos del mundo contemporáneo. Identidad y mentalidades. Madrid, CSIC, pp. 33-54. (2009): "La mujer sefardí ante sí misma y ante ellos: una lectura por las páginas de la Alborada (Sarajevo 1900-1901)". En: El Prezente. Studies in Sephardic Culture. Gender and Identity, n. ${ }^{\circ}$ 3, pp. 113-139.

(2006): Geografía Lingüística del Judeoespañol. Estudio sincrónico y diacrónico. Bern, Peter Lang.

Refael, Shmuel (2009): Un grito en el silencio. La poesía sobre el Holocausto en lengua sefardí: estudio y antología. Barcelona, Tirocinio.

Reyes Mate (2009): La herencia del olvido. Madrid, Errata Naturae.

Riaño López, Ana María, y Marcos Casquero, María del Carmen (2001): "Poesía contemporánea en lengua judeoespañola: Margalit Matitiahu y su obra". En: Estudios Humanísticos (Universidad de León). Filología, 23, pp. 141-172.

Romero, Elena (1992): La creación literaria en lengua sefardí. Madrid, Mapfre.

Romeu Ferré, Pilar (2008): "Sin memoria no hay avenir. Memorias escritas por mujeres sefardíes en los últimos 20 años". En: Revista de Dialectología y Tradiciones Populares, vol. LxIII, n. ${ }^{\circ}$ 2, pp. 101-120.

Rodrigue, Aron y Benbassa, Esther (2004): Historia de los judíos sefardíes: de Toledo a Salónica, Madrid: Abada.

Rodrigue, Aron (1990): French Jews, Turkish Jews. The Alliance Israélite Universelle and the Politics of Jewish Schooling in Turkey, 1860-1925. Bloomington, Indiana University Press.

(1983): "Jewish Society and Schooling in a Thracian Town: The Alliance Israélite Universelle in Demotica, 1897-1924". En: Jewish Social Studies, vol. 45, n. ${ }^{\circ} 3 / 4$, pp. 263-286.

Santa Puche, Salvador (2003): Libro de los testimonios: los sefardíes y el Holocausto. Barcelona, Sephardi Federation of Palm Beach County.

Schmid, Beatrice (2007): "De Salónica a Ladinokomunita. El judeoespañol desde los umbrales del siglo xx hasta la actualidad". En: Germán Colón y Lluís Gimeno Betí (eds.): Ecología lingüística i desaparició de llengües. Castelló de la Plana, Universitat Jaume I, pp. 9-33.

Séphiha, Haïm Vidal (1997): L'Agonie des Judéo-Espagnols. París, Entente.

Seroussi, Edwin (2003): "Archivists of Memory: Written Folksong Collections of TwentiethCentury Sephardi Women". En: Tullia Magrini (ed.): Music and Gender: Perspectives from the Mediterranean. Chicago-Londres, The University of Chicago Press, pp. 195214. 